\title{
Increased tooth brushing frequency is associated with reduced gingival pocket bacterial diversity in patients with intracranial aneurysms
}

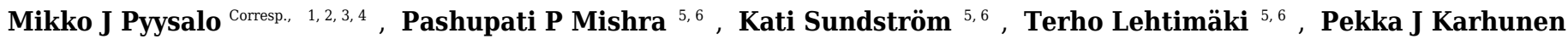 \\ 5,6 , Tanja Pessi ${ }^{4}$ \\ ${ }^{1}$ Department of Otorhinolaryngology, Faculty of Medicine and Health Technology, University of Tampere, Tampere, Finland \\ 2 Department of Oral and Maxillofacial diseases, Tampere University Hospital, Tampere, Finland \\ 3 Oral Health Services, City of Tampere, Tampere, Finland \\ 4 Department of Molecule Microbiology, Faculty of Medicine and Health Technology, University of Tampere, Tampere, Finland \\ 5 Faculty of Medicine and Health Technology and Finnish Cardiovascular Research Center, University of Tampere, Tampere, Finland \\ 6 Fimlab Laboratories Itd, Tampere, Finland \\ Corresponding Author: Mikko J Pyysalo \\ Email address: mikko.pyysalo@fimnet.fi
}

Objectives. The objective of this study was to investigate the association of tooth brushing frequency and bacterial communities of gingival crevicular fluid in patients subjected to preoperative dental examination prior to operative treatment for unruptured intracranial aneurysms. Methods.Gingival crevicular fluid samples were taken from their deepest gingival pocket from a series of hospitalized neurosurgical patients undergoing preoperative dental screening $(n=60)$. The patients were asked whether they brushed their teeth two times a day, once a day, or less than every day. Total bacterial DNA was isolated and the V3-V4 region of the 16S rRNA gene was amplificated. Sequencing was performed with Illumina's 16S metagenomic sequencing library preparation protocol and data were analyzed with QIIME (1.9.1) and R statistical software (3.3.2). Results.Bacterial diversity (Chaol index) in the crevicular fluid reduced along with reported tooth brushing frequency $[p=0.0002 ; R 2=34 \% ; p$ (adjusted with age and sex) $=0.09 ; R 2=11 \%$ ] showing that patients who reported brushing their teeth twice a day had the lowest bacterial diversity. According to the differential abundant analysis between the tooth brushing groups, tooth brushing associated with two phyla of fusobacteria $[p=0.0001 ; p=0.0007]$, and one bacteroidetes $[p=0.004]$ by reducing their amounts. Conclusions. Tooth brushing may reduce the gingival bacterial diversity and the abundance of periodontal bacteria maintaining oral health and preventing periodontitis, and thus it is highly recommended for neurosurgical patients. 
1 Increased tooth brushing frequency is associated with reduced gingival pocket bacterial

2 diversity in patients with intracranial aneurysms

3

4 Mikko Pyysalo ${ }^{1,2,3,4 *}$, Pashupati P. Mishra ${ }^{5,6}$, Kati Sundström ${ }^{5,6}$, Terho Lehtimäki ${ }^{5,6}$, Pekka J.

5 Karhunen ${ }^{5,6}$, Tanja Pessi ${ }^{4}$

$6{ }^{1}$ Department of Otorhinolaryngology, Faculty of Medicine and Health Technology, University

7 of Tampere, Tampere, Finland

$8 \quad{ }^{2}$ Department of Oral and Maxillofacial diseases, Tampere University Hospital, Tampere, Finland

$9{ }^{3}$ Oral Health Services, City of Tampere, Tampere, Finland; Tampere University Hospital,

${ }^{4}$ Department of Molecule Microbiology, Faculty of Medicine and Health Technology,

12 University of Tampere, Tampere, Finland

${ }^{5}$ Faculty of Medicine and Health Technology and Finnish Cardiovascular Research Center,

14 University of Tampere, Tampere, Finland

${ }^{6}$ Fimlab Laboratories Ltd, Tampere, Finland

16

Keywords: Bacterial DNA, biofilm(s), intracranial aneurysms, cardiovascular disease(s), fusobacteria, gingivitis, oral microbiome, preventive dentistry, 16S rRNA amplification.

*Corresponding author:

Mikko Pyysalo, Tampere University Hospital, Department of Oral and Maxillofacial diseases, P

21 O Box 2000, FIN-33521, Tampere, Finland

Fax: +358 331164105 Phone: +358 503439713

23 Email: mikko.pyysalo@,fimnet.fi 
Abstract

29 Objectives. The objective of this study was to investigate the association of tooth brushing

frequency and bacterial communities of gingival crevicular fluid in patients subjected to preoperative dental examination prior to operative treatment for unruptured intracranial aneurysms. Methods. Gingival crevicular fluid samples were taken from their deepest gingival pocket from a series of hospitalized neurosurgical patients undergoing preoperative dental screening $(n=60)$. The patients were asked whether they brushed their teeth two times a day, once a day, or less than every day. Total bacterial DNA was isolated and the V3-V4 region of the 16S rRNA gene was amplificated. Sequencing was performed with Illumina's 16S metagenomic sequencing library preparation protocol and data were analyzed with QIIME (1.9.1) and R statistical software (3.3.2). Results. Bacterial diversity (Chao1 index) in the crevicular fluid reduced along with reported tooth brushing frequency $[\mathrm{p}=0.0002 ; \mathrm{R} 2=34 \%$; $\mathrm{p}$ (adjusted with age and sex $=0.09 ; \mathrm{R} 2=11 \%$ ] showing that patients who reported brushing their teeth twice a day had the lowest bacterial diversity. According to the differential abundant analysis between the tooth brushing groups, tooth brushing associated with two phyla of fusobacteria $[p=0.0001 ; p=0.0007]$, and one bacteroidetes $[\mathrm{p}=0.004]$ by reducing their amounts. Conclusions. Tooth brushing may reduce the gingival bacterial diversity and the abundance of periodontal bacteria maintaining oral health and preventing periodontitis, and thus it is highly recommended for neurosurgical patients.

Introduction

The human oral cavity is considered healthy when the oral microflora is composed of indigenous bacteria and is properly balanced (Socransky and Haffajee 2002).

When dental plaque biofilm develops, it causes gingival inflammation that leads to marginal gingival swelling, the initiation of pocket formation, and the increased exudation of gingival crevicular fluid. If undisturbed, a mature biofilm develops in 1 to 2 weeks (Marsh and Martin 2009). Dental plaque biofilms are composed of microcolonies of bacterial cells that are non- 
55

56

57

58

59

60

61

62

63

64

65

66

67

68

69

70

71

72

73

74

75

76

77

78

79

80

81

82

83

randomly distributed in a shaped matrix or glycocalyx (Socransky and Haffajee 2002). Six bacterial groups are considered to be early biofilm colonizers: Actinomyces, a yellow complex comprising members of the genus Streptococcus, a green complex comprising the Capnocytophaga species, Actinobacillus actinomycetemcomitans serotype a, Eikenella corrodens and Campylobacter concisus, and a purple complex comprising Veillonella parvula and Actinomyces odontolyticus (Socransky et al. 1998). The growth of these six groups usually precedes the colonization of gram-negative orange (Fusobacterium nucleatum, Prevotella intermedia, Prevotella nigrescens, Parvimonas micra, Streptococcus constellatus, Eubacterium nodatum, Campylobacter showae, Campylobacter gracilis, Campylobacter rectus) and red (Porphyromonas gingivalis, Tannerella forsythia, Treponema denticola) complexes (Socransky et al. 1998). Recent studies have shown that the microbiota associated with periodontitis is much more complex than previously understood. Species from the genera Parvimonas, Filifactor, Dialister, Granilucatella, and Synergistes are found together with species belonging to the orange and red complexes (Colombo et al. 2009). Despite the active biofilm research, it still remains unclear which subgingival bacterial profiles are associated with the development of periodontal disease (Kononen and Muller 2014).

The use of cultivation independent 16S rRNA gene sequencing provides a powerful tool to analyze the complex microbiomes of environmental and clinical samples. Ai and co-workers (Ai et al. 2017) have suggested that alterations in the subgingival microbiome can be identified using metagenomic sequencing and used as a predictive marker of early periodontitis. The aim of this study was to investigate the impact of tooth brushing on the bacterial communities in the gingival crevicular fluid of patients undergoing preoperative dental screening prior to intracranial aneurysm treatment.

\section{Materials and methods}

In this study, the STROBE checklist for cohort studies was followed where applicable. This study was approved by the Ethics Committee of Pirkanmaa Hospital District (code R12217). Sixty patients subjected to preoperative dental screening due to a planned neurosurgical 
84 procedure for unruptured intracranial aneurysm were recruited to the study between September 852012 and December 2014. The inclusion criteria were having a saccular aneurysm, being aged 86 between 18 and 99 years, having the ability to give informed consent, and having a clinical 87 condition that allowed safe transportation to the radiological department situated in the same 88 building. An informed consent form approved by the hospital ethical committee was obtained 89 from all of the study patients. The exclusion criteria were requiring intensive care and being 90 unable to give informed consent.

During the acute hospitalization period for aneurysm treatment, an experienced oral and maxillofacial surgeon (MP) clinically investigated the patients' teeth. Periodontal probing was carried out using a standard WHO periodontal probe (LM 8-550B Si, LM-instruments Ltd, Parainen, Finland) with about $20 \mathrm{~g}$ force. Gingival pockets were measured from 6 sites of each tooth, and crevicular fluid samples for bacterial DNA analyses were taken from the deepest gingival pocket using a sterile blotting paper pin (Pearl Dent Co, Ho Chi Minh City, Vietnam). The samples were refrigerated at $-80{ }^{\circ} \mathrm{C}$ within 15 minutes. Patients were asked to report their tooth cleaning habits, i.e., how many times a day they cleaned their teeth.

DNA was isolated from the sample by using the standard protocol of QIAmp (Qiagen Ltd, California, USA). Gingival samples were amplified in duplicates using universal primers targeting the V3-V4 regions of the prokaryotic bacterial 16S rRNA gene: the forward primer with adapter was 341F TCG TCG GCA GCG TCA GAT GTG TAT AAG AGA CAG CCT ACG GGA GGC AGC AG (Muyzer et al. 1993), and the reverse primer with adapter was R806 GTC TCG TGG GCT CGG AGA TGT GTA TAA GAG ACA GGG ACT ACH VGG GTW TCT AAT (Caporaso et al. 2011). PCR reaction $(25 \mu \mathrm{l})$ was carried out with $2.5 \mu \mathrm{l}$ genomic DNA, 2x KAPA HiFi HotStart ReadyMix (Kapa Biosystems, USA), and the $0.2 \mathrm{mM}$ forward and $0.5 \mathrm{mM}$ reverse primer modified protocol of Takahashi and colleagues (Takahashi et al. 2014). Briefly, denaturation at $95^{\circ} \mathrm{C}$ for $3 \mathrm{~min}$, followed by 35 cycles of denaturation at $95{ }^{\circ} \mathrm{C}$ for $20 \mathrm{sec}$, annealing beginning at $65^{\circ} \mathrm{C}$ and ending at $55^{\circ} \mathrm{C}$ for $15 \mathrm{sec}$, and extension at $72{ }^{\circ} \mathrm{C}$ for $30 \mathrm{sec}$. The annealing temperature was lowered $1{ }^{\circ} \mathrm{C}$ every cycle until reaching $55^{\circ} \mathrm{C}$, which was then used for the remaining cycles. Final elongation was for $5 \mathrm{~min}$ at $72{ }^{\circ} \mathrm{C}$. Amplicon purification, second PCR, normalization, pooling, and sequencing was performed with Illumina's 16S metagenomic sequencing library preparation protocol (Illumina Inc., San Diego, California 
114 USA). MiSeq ${ }^{\circledR}$ Reagent Kit v3 for 600 sequencing cycles (Illumina Inc. San Diego, California) 115 was used for MiSeq library with a final concentration of $4 \mathrm{pM}$ and with $10 \%$ PhiX control. The 116 mock community was a DNA pool composed of Streptococcus mitis ATCC 49456,

117 Streptococcus sanguinis ATCC 10556, Streptococcus gorgonii ATCC 10558, Aggregatibacter 118 actinomycetemcomitans ATCC 700685, Porphyromonas gingivalis ATCC 33277, and E. coli 119 ATCC 25922 (LGC Standards, Teddington, Middlesex, UK).

120 Data analyses

121 The number of patients was 60. After amplification in duplicates, the quality of 63 (out of 120 122 possible) samples was sufficient for sequencing. After sequencing 34 samples from 23 patients 123 were of sufficient quality for the analyses. Eleven patient samples contained duplicates and 12 124 samples were single samples. The replicates were averaged before statistical analyses. To 125 investigate the gingival pocket bacterial communities of the study patients and to assess the alpha 126 diversity (chaol-index) (Chao 1984), we used the data analysis protocol presented in figure 1 in 127 which we show the use of the softwares QIIME (Caporaso et al. 2010), trimmomatic (Bolger et 128 al. 2014), phyloseq (McMurdie and Holmes 2013) and DESeq2 (Love et al. 2014). HOMD 129 (Chen et al. 2010) was used as a reference database. The regression analyses were made with and 130 without covariants (sex and age).

131 Statistical analyses were performed using R software (R Core Team (2013). R: A language and 132 environment $\mathrm{r}$ statistical computing. R Foundation for Statistical Computing, Vienna, Austria. 133 URL: http://www.R-project.org/).

137 The major phyla detected from the gingival pocket crevicular fluid samples were Firmicutes 138 (40.1\%), Bacteroidetes (28.0\%), and Fusobacteria (10.3\%). The major classes were Bacteroidia 139 (23.2\%), Negativicutes (21.3\%), Clostridia (11.2\%), and Fusobacteriia (10.3\%). 
140 The association of tooth brushing to the alpha diversity (chao1 index) level of gingival pocket

141 microbiome was calculated. The patients who reported brushing their teeth twice a day had the

142 lowest diversity, and those patients who brushed their teeth more seldom had the highest

143 diversity (Figure 2) ( $\mathrm{p}=0.0002 ; \mathrm{R} 2=34 \%$; Regression analysis). In addition, we also repeated

144 the same regression analysis with covariates age and sex $[p(\operatorname{adj})=0.09 ; R 2=11 \%]$. The relative

145 numbers of phyla divided into groups by the reported tooth brushing frequency are shown in

146 Figure 3. To see which bacterial taxa significantly differed between the groups, differential

147 abundant analysis (DAA) was performed (supplement 1). Two fusobacteria (both Leptotrichia -

148 genera) and one bacteroidetes (Prevotella -genus) reduced significantly along with teeth

149 brushing $[\mathrm{p}=0.0001, \mathrm{p}=0.0007$, and $\mathrm{p}=0.004$, respectively; Figure 4 and supplement 1$]$.

151

152

Discussion

153

154

155

156

157

158

159

160

161

162

163

164

165

166

167

168

The effect of tooth brushing on the alpha diversity level of the gingival pocket microbiome has previously been studied by Do Nascimento's (do Nascimento et al. 2015) and by Chen's (Chen et al. 2011) groups. Do Nascimento's group used the DNA checkerboard hybridization method to show that toothbrush bristles impregnated with silver nanoparticles reduced the total and individual genome count in the supra- and subgingival plaque biofilm after 4 weeks of brushing. However, chlorhexidine was not found to be effective in reducing the total genome counts in both supra- or subgingival biofilm after 4 weeks of brushing (do Nascimento et al. 2015). Chen and colleagues suggest that a favorable alteration in the microbial composition of dental plaque occurs $24 \mathrm{~h}$ after tooth brushing and professional dental prophylaxis. Importantly, an increase in bacterial diversity was observed after $24 \mathrm{~h}$ (Chen et al. 2011). In addition, Takeshita also showed that low bacterial diversity is associated with better oral health (Takeshita et al. 2016). Our results are in line with these previous findings.

In a healthy gingival pocket, there is a sparse streptococci dominating microbiome, into which different gram-negative rods (prevotella, fusobacteria, and porphyromonas) start colonizing if the plaque is left undisturbed (Marsh and Martin 2009). Takahashi and colleagues have proposed that fusobacteria are one of the key organisms in the early stage and create favorable conditions 
169 for other periodontal bacteria and subgingival inflammation through its allogenic factors, such as

$170 \mathrm{pH}$, oxygen levels, temperature, osmotic pressure and oxidation-reduction potential, and protein-

171 based nutrients. Fusobacteria are thus known to have a key role in pathogenic subgingival

172 biofilm formation and the binding of other colonizers (Takahashi et al. 2005, Kolenbrander et al.

173 2006). Subgingival biofilm may further induce destructive periodontitis and might also allow

174 bacteria to enter the bloodstream and cause infections elsewhere in the body (Socransky et al.

175 1998; Han et al. 2015). In this study, the increase in the relative number of fusobacteria was seen

176 to be associated with a low frequency of tooth brushing suggesting that tooth brushing may

177 disrupt the maturation process of the forming plaque biofilm.

178 There is a link between dental infections and cardiovascular diseases (Desvarieux et al. 2005;

179 Liljestrand et al. 2015; Buhlin et al. 2011; Tonetti and Van Dyke 2011). In our previous studies,

180 we detected both dental bacterial DNA and a bacterial driven inflammation in intracranial

181 aneurysm tissue samples (Pyysalo et al. 2013). Fusobacterium nucleatum was shown to be

182 among the most common bacteria in the aneurysm samples (Pyysalo et al. 2016). In this study,

183 we have shown that reported frequency of tooth brushing was associated with decreased bacterial

184 diversity and an abundance of fusobacteria in the gingival pocket crevicular fluid suggesting that

185 tooth brushing should be strongly recommended, especially for high-risk patients.

186

187 The use of molecular methods, such as 16S rRNA gene amplification based, whole genome

188 sequencing, and DNA-DNA hybridization, has revealed fundamental taxonomic changes

189 especially in anaerobic oral bacteria, such as fusobacteria, due to their slow/poor cultivation or

190 none cultivation properties. Although 16S rRNA gene sequencing is a novel technique in the

191 identification of cultivation-independent bacterial communities and remains the gold standard in

192 the determination of bacterial diversity, it has several limitations. For example, it is not the

193 recommended method in species-level identification due to the sequence similarity in the $16 \mathrm{~S}$

194 gene. Most bacterial strains have 97\% 16S rRNA sequence similarity, suggesting that 16S rRNA

195 gene sequencing provides genus identification in most cases ( $>90 \%$ ) but only $65 \%$ to $83 \%$

196 species information, leaving between $1 \%$ and $14 \%$ of the isolates unidentified. Moreover, the

197 obtained sequences are clustered and similarity threshold to cluster sequences into 'Operational 
198 Taxonomic Units' (OTU) is usually 97\%, and therefore limits the sequence data (Stackebrandt

199 and Ebers 2006; Janda and Abbott 2007; Petti 2007; Nguyen et al. 2016). Due to these

200 limitations, we identified OTUs into phylum and genus, not species levels. In addition, it would

201 be informative to conclude something on cause-effect in high-risk patients. The cross sectional

202 nature of the data in this study does not, however, support a direct cause-effect relationship. To

203 study the cause-effect of tooth brushing on the bacterial profile, we would need a longitudinal

204 study design. We also used sex and age as covariates in the regression model. The addition of

205 covariates to the model however worsens the model fit as shown by the higher P-value for

206 adjusted R squared. Due to the questionnaire based nature of the assessment of confounding

207 factors other than age and sex, we did not consider collecting other information reliable enough.

208 Other possible confounding factors could be dietary related factors, the delay between latest

209 tooth brushing and sampling, and the frequency of using mouthwash, for example. The results of

210 this study support the hypothesis that the increase in tooth brushing frequency has a positive

211 effect on subgingival pocket microbiome, which could be considered as a long term effect. Short

212 term effects on supragingival plaque can be caused by dietary factors, such as high sugar intake

213 and occasional use of mouthwash. However if tooth brushing frequency remains constantly high

214 enough, the bacterial plaque has a limited chance to invade the subgingival space.

\section{Conclusion}

216 Subgingival microbiota plays an important role in the pathogenesis of gingival inflammation.

217 The collection of non-invasive gingival crevicular fluid (GCF) and the identification of bacterial

218 communities in it may provide an informative diagnostic tool to evaluate the microbiota

219 environment associated with deep pocket depths and severe gingival inflammation in patients

220 undergoing preoperative dental screening. Subgingival bacterial diversity and the relative

221 abundance of fusobacteria in GCF may be reduced by active tooth brushing. 
225

226

227

228

229

230

231

232

233

234

235

236

237

238

239

240

241

242

243

244

245

246

247

248

249

250

251

252

253

254

255

Ai D, Huang R, Wen J, Li C, Zhu J, Xia LC. 2017. Integrated metagenomic data analysis demonstrates that a loss of diversity in oral microbiota is associated with periodontitis. BMC Genomics. 18(Suppl 1):1041. DOI: 10.1186/s12864-016-3254-5.

Buhlin K, Mäntylä P, Paju S, Peltola JS, Nieminen MS, Sinisalo J, Pussinen PJ. 2011. Periodontitis is associated with angiographically verified coronary artery disease. J Clin Periodontol 38(11):1007-1014. DOI: 10.1111/j.1600-051X.2011.01775.x.

Bolger AM, Lohse M, Usadel B. 2014. Trimmomatic: a flexible trimmer for Illumina sequence data. Bioinformatics 30(15):2114-2120.

Caporaso JG, Lauber CL, Walters WA, Berg-Lyons D, Lozupone CA, Turnbaugh PJ, Fierer N, Knight R. 2011. Global patterns of 16S rRNA diversity at a depth of millions of sequences per sample. Proc Natl Acad Sci U S A 108 Suppl 1:4516-4522. DOI: 10.1073/pnas.1000080107.

Caporaso JG, Kuczynski J, Stombaugh J, Bittinger K, Bushman FD, Costello EK, Fierer N, Peña AG, Goodrich JK, Gordon JI, Huttley GA, Kelley ST, Knights D, Koenig JE, Ley RE, Lozupone CA, McDonald D, Muegge BD, Pirrung M, Reeder J, Sevinsky JR, Turnbaugh PJ, Walters WA, Widmann J, Yatsunenko T, Zaneveld J, Knight R. 2010. QIIME allows analysis of highthroughput community sequencing data. Nat Methods. 7(5):335-336. doi: 10.1038/nmeth.f.303.

Chao, A. 1984. Nonparametric estimation of the number of classes in a population. Scandinavian Journal of statistics, 265-270.

Chen T, Yu WH, Izard J, Baranova OV, Lakshmanan A, Dewhirst FE 2010. The Human Oral Microbiome Database: a web accessible resource for investigating oral microbe taxonomic and genomic information. Database, 2010.

Chen Z, Trivedi HM, Chhun N, Barnes VM, Saxena D, Xu T, Li Y. 2011. Using DGGE and 16S rRNA gene sequence analysis to evaluate changes in oral bacterial composition. Chin J Dent Res. 14(2):95-103. 
256 Colombo AP, Boches SK, Cotton SL, Goodson JM, Kent R, Haffajee AD, Socransky SS, 257 Hasturk H, Van Dyke TE, Dewhirst F, Paster BJ. 2009. Comparisons of subgingival microbial 258 profiles of refractory periodontitis, severe periodontitis, and periodontal health using the human 259 260 oral microbe identification microarray. J Periodontol 80(9):1421-1432. DOI: 10.1902/jop.2009.090185.

261

Kononen E, Muller HP. 2014. Microbiology of aggressive periodontitis. Periodontol 2000

Janda JM, Abbott SL 2007. 16S rRNA Gene Sequencing for Bacterial Identification in the Diagnostic Laboratory: Pluses, Perils, and Pitfalls. J Clin Microbiol. 45(9): 2761-2764.

do Nascimento C, Paulo D, Pita M, Pedrazzi V, de Albuquerque R Jr. 2015. Microbial diversity of the supra- and subgingival biofilm of healthy individuals after brushing with chlorhexidine- or silver-coated toothbrush bristles. Canadian Journal of Microbiology 61(2). 112-123. DOI: 10.1139/cjm-2014-0565.

Kolenbrander PE, Palmer RJ Jr, Rickard AH, Jakubovics NS, Chalmers NI, Diaz PI. 2006. Bacterial interactions and successions during plaque development. Periodontol 2000. 42:47-79.

280

281

282

283

284

285

286

287

288

289

290

Desvarieux M, Demmer RT, Rundek T, Boden-Albala B, Jacobs DR, Sacco RL, Papapanou PN. 2005. Periodontal microbiota and carotid intima-media thickness: the Oral Infections and Vascular Disease Epidemiology Study (INVEST). Circulation 111(5):576-582.

65:46-78. DOI: 10.1111/prd.12016.

Liljestrand JM, Havulinna AS, Paju S, Männistö S, Salomaa V, Pussinen PJ. 2015. Missing teeth predict incident cardiovascular events, diabetes and death. J Dent Res 94(8):1055-1062. DOI: $10.1177 / 0022034515586352$.

Love MI, Huber W, Anders S. 2014. Moderated estimation of fold change and dispersion for RNA-seq data with DESeq2. Genome biology, 15(12), 550.

Marsh PD, Martin MV. Oral Microbiology, 5th ed. Edinburgh, London, New York, Oxford, Philadelphia, St Louis, Sydney, Toronto: Churchill Livingstone Elsevier, 2009. 
291

292

293

294

295

296

297

298

299

300

301

302

303

304

305

306

307

308

309

310

311

312

313

314

315

316

317

318

319

320

321

322

323

324

325

McMurdie, PJ, Holmes S. 2013. phyloseq: an R package for reproducible interactive analysis and graphics of microbiome census data. PloS one, 8(4), e61217.

Muyzer G, de Waal EC, Uitterlinden AG. 1993. Profiling of complex microbial populations by denaturing gradient gel electrophoresis analysis of polymerase chain reaction-amplified genes coding for 16S rRNA. Appl Environ Microbiol 59(3):695-700.

Nguyen NP, Warnow T, Pop M, White B. 2016. A perspective on 16S rRNA operational taxonomic unit clustering using sequence similarity. NPJ Biofilms Microbiomes. 20(2):16004. DOI: 10.1038/npjbiofilms.2016.4.

Petti, C. A. 2007. Detection and identification of microorganisms by gene amplification and sequencing. Clin. Infect. Dis. 44:1108-1114.

Pyysalo MJ, Pyysalo LM, Pessi T, Karhunen PJ, Öhman JE. 2013. The connection between ruptured cerebral aneurysms and odontogenic bacteria. J Neurol Neurosurg Psychiatry. 84(11):1214-1218. DOI: 10.1136/jnnp-2012-304635.

Pyysalo MJ, Pyysalo LM, Pessi T, Karhunen PJ, Lehtimäki T, Oksala N, Öhman J. 2016. Bacterial DNA findings in ruptured and unruptured intracranial aneurysms. Acta Odontol Scand. 74(4):315-320. DOI: 10.3109/00016357.2015.1130854.

Takahashi N. Microbial ecosystem in the oral cavity: metabolic diversity in an ecological niche and its relationship with oral diseases. 2005. Int Congr Ser.;1284:103-112

Takahashi S, Tomita J, Nishioka K, Hisada T. Nishijima M. 2014. Development of a prokaryotic universal primer for simultaneous analysis of Bacteria and Archaea using next-generation sequencing. PloS one, 9(8), e105592. DOI: 10.1371/journal.pone.0105592.

Takeshita T, Kageyama S, Furuta M, Tsuboi H, Takeuchi K, Shibata Y, Shimazaki Y, Akifusa S, Ninomiya T, Kiyohara Y, Yamashita Y. 2016. Bacterial diversity in saliva and oral healthrelated conditions: the Hisayama Study. Sci Rep. 6:22164. DOI: 10.1038/srep22164.

Tonetti MS, Van Dyke TE. 2013. Periodontitis and atherosclerotic cardiovascular disease consensus report of the Joint EFP/AAP Workshop on Periodontitis and Systemic Disease. J Clin Periodontol 40 (Suppl 14):S24-S29. DOI: 10.1902/jop.2013.1340019. 
327 Socransky SS, Haffajee AD, Cugini MA, Smith C, Kent RL Jr. 1998. Microbial complexes in 328 subgingival plaque. J Clin Periodontol 25(2):134-144.

330 Socransky SS, Haffajee AD. 2002. Dental biofilms: difficult therapeutic targets. Periodontol $331200028: 12-55$.

332

333 Socransky SS, Haffajee AD. 2005. Periodontal microbial ecology. Periodontol 2000. 38:135334187.

335

336 Stackebrandt, E. Ebers, J. 2006 Taxonomic parameters revisited: tarnished gold standards.

337 Microbiology Today 33(4); 152-155

338

339 Figure legends

340 Fig 1: Data analysis protocol. Sequences were clustered into the operational taxonomic units 341 (OTUs) using 97\% similarity threshold.

342 Fig 2: The effect of tooth brushing to the alpha diversity (chao1 index) level of gingival pocket 343 microbiome.

344 Fig 3: The relative abundance of taxa at phyla and class levels divided into groups by tooth 345 brushing frequency

346 Fig 4: Three most significantly differing Operational taxonomic units (OTUs) between the tooth 347 brushing frequency groups.

348 Fusobacteria1: genus1: Leptotrichia

349 Fusobacteria2: genus2: Leptotrichia

350 Bacteroidetes: genus: Prevotella 


\section{Figure 1 (on next page)}

Data analysis protocol

Sequences were clustered into the operational taxonomic units (OTUs) using $97 \%$ similarity threshold. 
1. Quality Control (QC)
a. QC check with FastQC
b. Trim primers
c. Trim low quality bases (phred score $<20$ )
d. Remove short sequences $(<200$ base pairs)
e. QC check with FastQC

2. Data preparation: Prepare input files for QIIME

a. Merge paired ends using fasta-join in QIIME

b. Generate QIIME sequence and map file

3. Contamination filtration
a. Remove chimera using usearch61 implemented in QIIME
b. Remove mitochondrial, chloroplast, archaea and eucaryotic sequences using mothur functionalities
c. Remove bacterial contamination using control and replicate sequences

4. OTU clustering

OTU clustering (97\% sequence similarity) with uclust in QIIME

5. Taxonomy assignment

Assign taxonomy to representative OTU sequences using Human Oral Microbiome Database (HOMD) with uclust method implemented in QIIME

6. Downstream analysis

a. Alpha diversity analysis with phyloseq

b. Differential abundance analysis (DAA) using DESeq2 implemented in phyloseq 
Figure 2 (on next page)

The effect of tooth brushing to the alpha diversity (chaol index) level of gingival pocket microbiome 

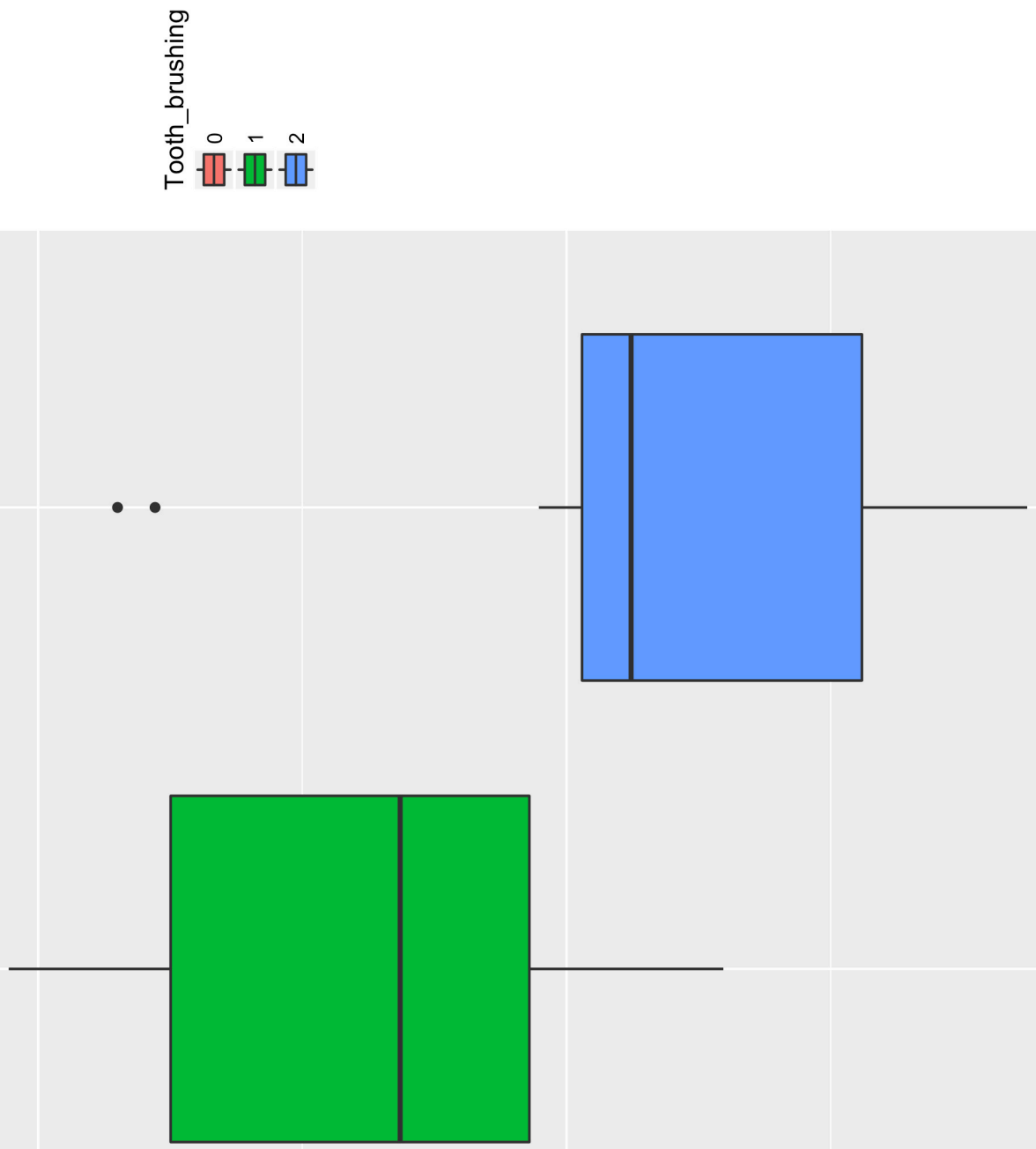
Figure 3

The relative abundance of taxa at phyla and class levels divided into groups by tooth brushing frequency 


Figure 4(on next page)

Three most significantly differing Operational taxonomic units (OTUs) between the tooth brushing frequency groups

Fusobacteria1: genus1: Leptotrichia

Fusobacteria2: genus2: Leptotrichia

Bacteroidetes: genus: Prevotella 

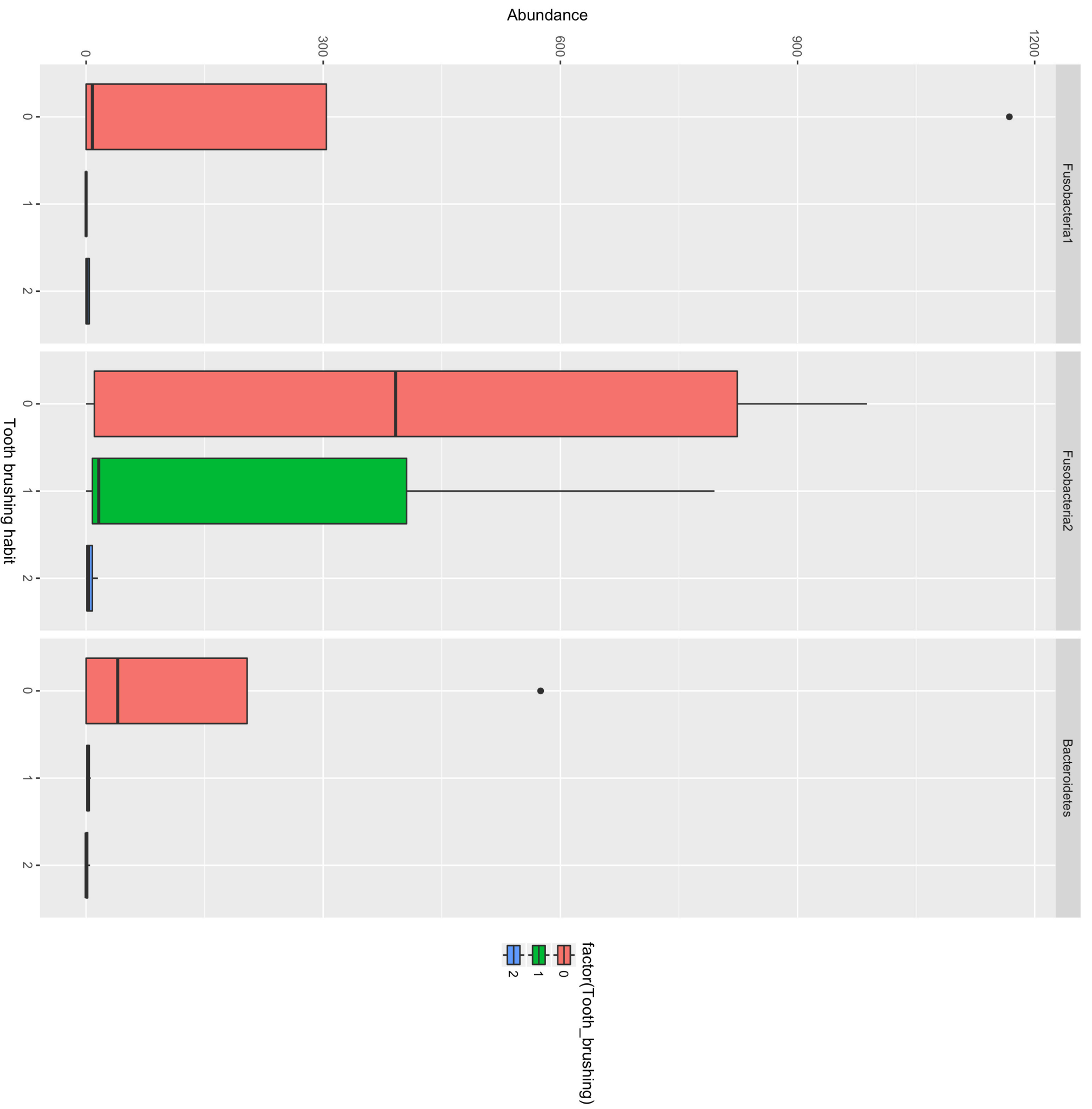